\title{
Prospects of quantum information processing with atoms
}

\author{
Peter Zoller
}

Received: 26 August 2011 / Published online: 20 September 2011

(C) Springer Science+Business Media, LLC 2011

Quantum information, and particular questions of implementing quantum information processing, is now almost two decades old, and has developed into a mature field of physics. In the first decade many of the conceptual foundations of the field of quantum information were laid down, many of the basic theoretical proposals for implementing quantum computing and quantum communications with various physical systems were formulated, and a first generation of experimental demonstrations of some of the basic building blocks and protocols of QIPC were shown in the lab. Today we are in a phase where the experimental challenge is not only to achieve high fidelity in operations, but to scale up systems both in the number of qubits and in the complexity of the quantum operations towards fault tolerant quantum computing. Progress during the last few years has been remarkable, and exceeded our early dreams, although on the scale of futuristic goals and hopes of QIPC this remains a first step.

The requirements of implementing QIPC include the manipulation of many particle quantum systems on the level of single quanta, the ability to prepare and read out quantum states with high fidelity, while achieving complete isolation from the environment. Quantum optical systems of trapped ions, atoms, molecules and photons, as in CQED, have always had a leading role in achieving these goals. The rapid progress in achieving quantum control in many particle physics happens, of course, in a much broader context, and solid state systems such as superconducting qubits, quantum dots and NV centers, as well as circuit QED and nanomechanics are becoming serious contenders for atomic systems.

The present issue focuses on quantum information processing with neutral atoms and molecules. What do we see as interesting future directions and possibilities?

\footnotetext{
P. Zoller $(\varangle)$

Institute for Theoretical Physics, University of Innsbruck, Institute for Quantum Optics, and Quantum Information of the Austrian Academy of Sciences, Innsbruck, Austria e-mail: peter.zoller@uibk.ac.at
} 
Until recently, various quantum optical systems had clear associations with different corners in the world of QIPC, e.g. trapped ions were synonymous with general purpose quantum computing, while atoms were associated mainly with implementing quantum simulation. As we argue below, based on recent experimental achievements this situation has changed, opening new and promising directions of development and challenges for the coming years.

In the past, systems of trapped ions were representative of a bottom up approach where for a small number of qubits all the requirements of general purpose quantum computing were fulfilled. This includes a complete toolbox of coherent single qubit and entangling gate manipulations with a fidelity better than the percent level, and readout on the single qubit level. A challenge for ions has been the development of a scalable architecture. However, first steps in this direction have been taken, for example, by shuttling ions between a storage (memory) zone and a processor unit, where quantum gates on a small number of qubits are performed. In general purpose quantum computing, a taunting challenge is, of course, the requirement of implementing fault tolerant quantum computing, although again first demonstrations of error correction have recently been reported.

On the other hand, systems of atoms and molecules have so far been associated mainly with a top down approach with focus on quantum simulation. In particular, a large number of atoms can be loaded with exactly one atom per site in an optical lattice in two and three spatial dimensions as a Mott insulator, representing an a priori scalable system of qubits. However, until recently, in these systems only global control of qubits was available, making these systems ideal candidates for simulation of (translationally invariant) spin models, as special purpose quantum processors. In fact, remarkable experimental achievements have been reported in emulating Hubbard and spin physics of strongly interacting systems with atoms and molecules in optical lattices.

This situation has changed, however. Recent trapped ion experiments have reported on small scale quantum simulations of the dynamics of frustrated spin systems including long range interactions. In particular, the possibility of high fidelity entangling gates has opened the door to digital quantum simulation (as opposed to an analog emulation), where the time evolution of the many body system is approximated by a sequence of a very large number of gates. On the other hand, on the neutral atom side recent technical achievements of addressing and thus manipulating single atoms in optical lattices and the development of single atom traps and trap arrays, as with atom chips, promise an a priori scalable (general purpose) quantum processor with atoms, where entangling gates between a given pair of qubits can be implemented with long-range Rydberg interactions, or possibly entangling atoms by moving around a set of messenger atoms in combination with local collisional interactions. A challenge for neutral atoms is, of course, to catch up with ions on the level of fidelity in performing gate operations. A long term vision is not only to build a quantum processor, but to connect these processors in quantum networks with applications in quantum communications and as a way to scale up quantum computers. Recent experiments in CQED with single atoms and atomic ensembles have demonstrated the light—atom interfaces as the basic building block for such a network.

What should the medium term goals for QIPC be? It is essential that we have demonstrations of non-trivial experimental applications or examples of quantum information 
processing within the next few years involving large scale entanglement. Such nontrivial demonstrations are essential in light of the visions presented as motivation for QIPC during the last two decades; by non-trivial we mean applications involving a many particle system and entanglement, which (i) are of interest, and hopefully useful, to a community broader than the quantum information community per se, and (ii) on a level not achievable by any classical device. It seems that the present and certainly the coming generation of experiments with quantum optical systems, and in particular also neutral atoms and molecules, will provide a solid and promising basis for such demonstrations.

What could these demonstrations be? It is an intrinsic feature that progress in quantum engineering involves an increasingly technological component. It is essential, however, that -in particular since true large scale quantum computing is far in the future-each stage of technological development is a stepping stone, and is illustrated and associated with new conceptual insights and advances in physics. In this sense Feynman's quantum simulation as non-trivial many body physics has played and will play a special role. The challenge will be to perform experiments, which involve experimentally demonstrated large scale entanglement which cannot be represented on a classical computer. For ion trap experiments this will require scaling the present experiments to a few tens of qubits, which may be in reach in coming years. In this context it is particularly exciting to see that a quantitative comparison of elaborate and rigorous calculations with recent experiments on quench dynamics in Munich, reveal that the long time dynamics seen experimentally cannot be computed (classically) because of growth of entanglement. Besides such fundamental demonstrations, quantum simulation is interesting as a novel form of controlled and engineered condensed matter platform for novel insight into the physics of strongly correlated systems. By simulating exotic quantum phases, we will also realize such new quantum materials in the laboratory. The exotic phases might support novel kind of excitations, as anyons for topological quantum computing. This may provide the starting point of a new era in quantum optics, where we not only learn how to manipulate atoms, ions, molecules and photons on a single quantum level, but even design our own new elementary objects.

While we have discussed quantum computing from the point of view of the quantum logic network model, there must be room for conceptually new developments in quantum information and associated experiments, an example being adiabatic quantum computing or the recently discussed dissipative quantum computing.

Finally, many of the applications will, as in the past, be in quantum sensing and precision measurement.

Quantum optics has always been a field driven by experimental advances. In this sense new systems become available with their unique features and potential for QIPC. A recent example is Alkaline Earth atoms, which provide the best neutral atomic optical clocks, and thus is also a promising novel natural starting point for implementing QIPC. A second example is given by hybrid systems as combinations of atomic and solid state systems, and as an interface between these two worlds.

What exists today in the laboratory exceeds the dreams and hopes we had ten years ago in implementing QIPC. We might wonder where we will be ten years from now. 